\title{
Low profilin 1 serum levels are associated with diabetes, family history and multivessel lesions in patients with coronary artery disease
}

\author{
Elżbieta Paszek ${ }^{1}$, Wojciech Zajdel ${ }^{1}$, Krzysztof Plens² $^{2}$ Krzysztof Żmudka³ ${ }^{3}$ Jacek Legutko ${ }^{3}$, Paweł Kleczyński \\ ${ }^{1}$ Clinical Department of Interventional Cardiology, John Paul II Hospital, Krakow, Poland \\ ${ }^{2}$ KCRI, Krakow, Poland \\ ${ }^{3}$ Department of Interventional Cardiology, Institute of Cardiology, Jagiellonian University Medical College, John Paul II Hospital, Krakow, \\ Poland
}

Adv Interv Cardiol 2021; 17, 3 (65): 305-308

DOI: https://doi.org/10.5114/aic.2021.109159

\section{Introduction}

Atherosclerosis, underlying coronary artery disease (CAD), is best characterized as a low-grade inflammatory condition, where cholesterol particles - mostly oxidized low-density lipoproteins (ox-LDL) - infiltrating the vessel wall act as an antigen and initiate an inflammatory response [1]. This approach has been explored for many years now but does not allow for a full understanding of the disease. Recently, the actin cytoskeleton and associated regulatory proteins have been gaining attention as contributors to the pathophysiology of CAD [2]. Among these, profilin 1 ( $P f n 1$ ) is an emerging player in the field $[3,4]$. Research showed that Pfn 1 was overexpressed in atherosclerotic lesions in comparison to the healthy vessel wall [5]. In mice a deletion of one copy of the PFN1 gene increased the production of nitric oxide, lowered the expression of adhesion molecules and hindered macrophage infiltration, thereby protecting against atherosclerosis [6]. In diabetic atherosclerosis models, Pfn 1 was overexpressed in endothelial cells exposed to advanced glycation end-products, accompanied by hallmarks of endothelial dysfunction [7]. Pfn1 was also implicated in the pathophysiology of acute coronary syndromes. It was found within thrombi retrieved from culprit arteries and correlated with symptom duration, as well as final Thrombolysis in Myocardial Infarction flow [8, 9].

So far there has been very little research on Pfn 1 in humans, and it has never been assessed in relation to atherosclerosis risk factors or the severity of atherosclerosis estimated by coronary angiography.

\section{Aim}

To assess serum concentrations of Pfn 1 in relation to risk factors and extent of coronary artery disease.

\section{Material and methods}

The study was approved by the institutional ethical board (decision no. 122.6120.270.2015; $17^{\text {th }}$ December 2015) and was conducted in accordance with the principles listed in the 1964 Declaration of Helsinki. We enrolled consecutive patients with CAD qualified for coronary angiography, who presented with chronic coronary syndrome (CCS) or myocardial infarction (MI). Exclusion criteria were as follows: an $\mathrm{Ml}$ other than type 1 , an $\mathrm{MI}$ with symptom duration of more than $24 \mathrm{~h}$, prior coronary artery bypass grafting, active inflammation, thrombosis in vessels other than coronary arteries, an active neoplasm (diagnosed and/or treated within the last year), a stroke (ischemic or hemorrhagic) within 3 months prior to admission, known pro-thrombotic conditions, chronic anticoagulation, and estimated glomerular filtration rate $<30 \mathrm{ml} / \mathrm{min} / 1.73 \mathrm{~m}^{2}$.

We checked patients for the presence of typical atherosclerosis risk factors and co-existing conditions. Hypertension was defined as a blood pressure $\geq 140 / 90 \mathrm{~mm} \mathrm{Hg}$ measured on two separate occasions, a history of hypertension or chronic use of antihypertensive drugs. Hypercholesterolemia was defined as total cholesterol of more than $5.0 \mathrm{mmol} / \mathrm{l}$ and/or low-density lipoprotein $(\mathrm{LDL})$-cholesterol of more than $3.0 \mathrm{mmol} / \mathrm{l}$, or in patients with lipid-lowering pharmacotherapy. Diabetes mellitus was coded when the patient had a prior diagnosis or if

\section{Corresponding author:}

Elżbieta Paszek MD, Clinical Department of Interventional Cardiology, John Paul II Hospital, 80 Prądnicka St, 31-202 Krakow, Poland, phone: +48 886886 352, e-mail: elzbieta.m.paszek@gmail.com

Received: 25.06.2021, accepted: 22.07.2021. 
glycemia and/or glycated hemoglobin $\left(\mathrm{HbA}_{1 \mathrm{c}}\right)$ concentrations met the definitions included in the appropriate guidelines [10]. Active smoking was defined as smoking 10 or more cigarettes/day within the last 6 months prior to admission. Obesity was coded when a patient's body mass index was $30 \mathrm{~kg} / \mathrm{m}^{2}$ or above. A family history of CAD was coded when a family member in the first degree (parents, siblings, children) experienced myocardial revascularization, $\mathrm{Ml}$ and/or cardiovascular death before the age of 55 for men and 65 for women.

Multivessel disease was defined as significant stenosis ( $\geq 50 \%$ for left main and $70 \%$ for other vessels) of three or more epicardial coronary arteries with a diameter of at least $2 \mathrm{~mm}$ [11].

The severity of coronary atherosclerosis was also assessed using the Gensini Score [12]. Even though it is widely used in clinical practice, we did not assess the SYNTAX score, as it is a measure of lesion complexity more than an indicator of atherosclerotic burden. We analyzed whether the patient had experienced an acute coronary syndrome or percutaneous coronary angioplasty in the past. Moreover, we searched for correlations between plasma Pfn1 and standard laboratory parameters.

\section{Profilin 1 concentration measurement}

A $2.6 \mathrm{ml}$ sample of venous blood was collected into an EDTA-coated tube from each patient on admission, prior to the procedure. The samples were centrifuged at $1600 \mathrm{~g}$ for $10 \mathrm{~min}$. The supernatant was collected and stored at $-80^{\circ} \mathrm{C}$, until further use. Pfn 1 concentration was determined using the enzyme-linked immunosorbent assay (ELISA). We used the human profilin 1 SEC233Hu ELISA kit (Cloud-Clone Corp., Houston, USA) with a detection range of $78-5000 \mathrm{pg} / \mathrm{ml}$. This is a test employing the biotin-avidin-horseradish peroxidase system. $100 \mu$ of undiluted serum sample was inserted per well. The spectrophotometric reading was performed at $450 \mathrm{~nm}$, immediately after using the stop solution. A calibration curve was prepared by measuring the absorbance at the

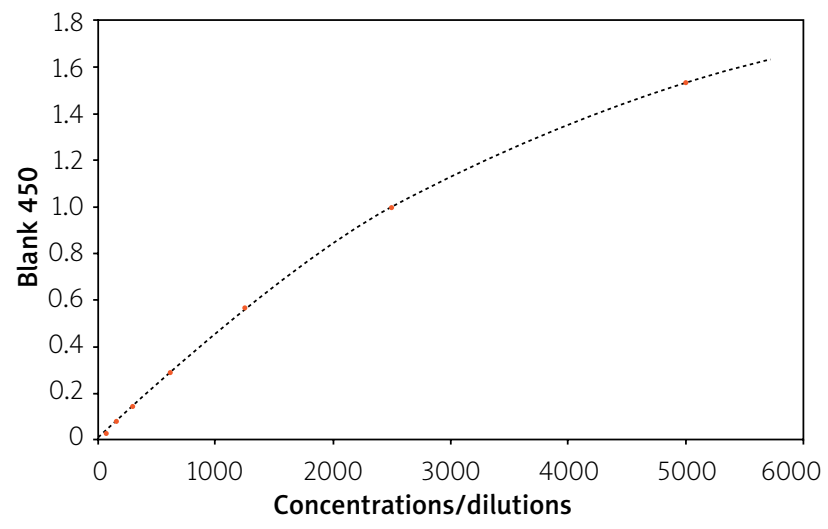

Figure 1. Standard curve for the ELISA test used to assess profilin 1 concentrations same wavelength using serial dilutions of a given standard [13]. The calibration curve is shown in Figure 1.

\section{Statistical analysis}

Continuous variables were checked for normal distribution by the Shapiro-Wilk test. Pfn 1 concentrations were compared using the Mann-Whitney test and presented as median and $25-75 \%$ interquartile range (IQR), as the data did not fulfil the assumption of normality of distribution. The Spearman correlation test was used to compare two numerical variables. The level of significance was assumed at $\alpha=0.05$.

\section{Results}

We included a hundred patients with CAD admitted for coronary angiography: 65 with $\mathrm{MI}$ and 35 with CCS. There was no difference in serum Pfn1 levels between patients with $\mathrm{MI}$ and CCS. We found that median Pfn1 concentrations were significantly lower in patients with diabetes $(p=0.008)$, family history of CAD $(p=0.043)$ and multivessel disease $(p=0.018)$. We did not find a correlation between Pfn 1 concentration and Gensini score (rho $=0.086 ; p=0.779$ ). For the association between Pfn 1 and LDL we obtained values of rho $=0.198$, $p=0.056$; and for Pfn 1 and C-reactive protein (CRP), rho = $0.309, p=0.076$. The results are shown in Tables I and II.

\section{Discussion}

This is the first study showing that in patients with symptomatic CAD, serum Pfn1 levels are lower in patients with diabetes, family history of CAD and multivessel disease.

\section{Pfn1 and diabetes}

Previous studies using animal and in vitro models suggested detrimental effects of Pfn 1 in diabetes-associated atherosclerosis. Li et al. found that exposing human endothelial cells to advanced glycation end-products led to an increase in Pfn1 expression and cytoskeletal rearrangement. This was accompanied by a decrease in nitric oxide release, as well as a rise in intercellular adhesion molecule 1 and asymmetric dimethylarginine [7]. Pfn1 expression was higher in aortic endothelial cells of diabetic rats, compared with specimens without diabetes. Inducing Pfn 1 expression in these cells led to a rise in intercellular adhesion molecule 1 expression, as well as apoptosis. Importantly, endothelial cells treated with LDL and ox-LDL showed higher Pfn1 expression [14].

Our results seem to be counterintuitive in the context of the above. This may be due to the fact that serum Pfn 1 does not have to reflect its intracellular levels. Another explanation could be that serum Pfn1 may undergo carbonylation, which is a common mechanism of posttranslational modifications under persistent hyperglycemia [15]. Carbonylation is known to alter protein structure and 
Table I. Profilin 1 serum concentrations with regard to known atherosclerosis risk factors and severity of coronary artery disease

\begin{tabular}{lccc} 
Factor & $\begin{array}{c}\text { Factor present; } \\
\text { Pfn1 concentration [pg/ml] } \\
\text { Median (IQR) }\end{array}$ & $\begin{array}{c}\text { Factor absent; } \\
\text { Pfn1 concentration [pg/ml] } \\
\text { Median (IQR) }\end{array}$ & $P$-value \\
\hline Myocardial infarction & $784.37(652.17-1523.32)$ & $764.70(657.61-1019.87)$ & 0.614 \\
\hline Gender: male & $767.41(651.14-1305.00)$ & $789.83(708.77-1265.05)$ & 0.730 \\
\hline Hypertension & $767.83(655.33-1244.11)$ & $829.91(650.00-1528.55)$ & 0.849 \\
\hline Dyslipidemia & $781.38(650.17-1230.18)$ & $760.15(693.07-1534.47)$ & 0.831 \\
\hline Diabetes & $713.91(579.83-873.78)$ & $834.84(709.23-1503.74)$ & 0.008 \\
\hline Smoking & $768.26(681.48-1042.74)$ & $764.70(624.74-1305.00)$ & 0.789 \\
\hline Obesity & $739.99(660.15-924.62)$ & $844.14(653.77-1501.78)$ & 0.061 \\
\hline Family history of CAD & $739.99(634.08-847.63)$ & $814.29(656.85-1472.12)$ & 0.043 \\
\hline Multivessel disease & $714.49(583.16-947.87)$ & $793.02(706.76-1523.32)$ & 0.018 \\
\hline Prior myocardial infarction & $762.34(591.22-992.47)$ & $781.38(681.48-1499.83)$ & 0.224 \\
\hline Prior PCl & $756.42(644.68-1124.77)$ & $784.37(663.58-1335.98)$ & 0.591
\end{tabular}

$C A D$ - coronary artery disease (definition in text), $\mathrm{PCl}$ - percutaneous coronary intervention.

function [16]. This could lead to weaker binding with the ELISA antibody and lower detection. To confirm this, a larger study is needed focusing on Pfn 1 structure under chronic hyperglycemia as well as on markers of glycemia control and duration of the disease.

\section{Pfn 1 and family history of CAD}

We found a lower Pfn 1 concentration in patients with a family history of CAD. Although we assessed the protein and not the gene itself, it cannot be ruled out that genetic variants of the PFN1 gene leading to structural and functional changes are relevant in CAD. Lower ELISA readings could be a result of three phenomena: instability of a mutant protein, a tendency to form aggregates or a conformational change leading to a weaker interaction with the antibody employed in the test. In fact, Pfn 1 mutations have been reported in other human diseases, foremostly in amyotrophic lateral sclerosis [17]. The best characterized mutations include C71G, M114T, G118V and E117G. All of these mutants have an altered tertiary structure and easily form aggregates (with the C71G being the most prone to aggregation) [18], while M114T causes conformational changes to the core of the protein, impairing its stability [19]. Taking into account that this is a preliminary result, it may be reasonable to search for an association between genetic variants of the PFN-1 gene and cardiovascular outcomes.

\section{Severity of CAD}

The relationship between $\mathrm{Pfn} 1$ concentration and the extent of coronary lesions is unclear. We found no association with a history of $\mathrm{MI}$ or percutaneous coronary intervention $(\mathrm{PCl})$. We found that patients with multivessel disease had lower serum profilin, but we did not confirm
Table II. Spearman's correlations between serum profilin 1 concentrations and standard laboratory results

\begin{tabular}{lcc}
\multirow{2}{*}{ Parameter } & \multicolumn{2}{c}{ Profilin 1 serum concentration } \\
\cline { 2 - 3 } & Spearman's rho & P-value \\
\hline Red blood count & -0.063 & 0.538 \\
\hline White blood count & 0.022 & 0.827 \\
\hline Neutrophils & -0.014 & 0.893 \\
\hline Lymphocytes & 0.143 & 0.156 \\
\hline Platelets & 0.007 & 0.942 \\
\hline $\begin{array}{l}\text { Glomerular filtration } \\
\text { rate }\end{array}$ & -0.004 & 0.971 \\
\hline Total cholesterol & 0.144 & 0.168 \\
\hline $\begin{array}{l}\text { Low-density lipopro- } \\
\text { tein cholesterol }\end{array}$ & 0.198 & 0.056 \\
\hline C-reactive protein & 0.309 & 0.076
\end{tabular}

this result using the Gensini method. This result requires further studies.

\section{Basic laboratory results}

Although there were non-significant associations between Pfn 1 and LDL $(p=0.056)$ and CRP $(p=0.076)$, these results require further evaluation in a larger group of patients.

\section{Study limitations}

The small sample size and data overlap between the compared groups does not allow us to draw clinically applicable conclusions at this point. Also, due to the fact that we analyzed coronary angiograms, we did not include healthy volunteers as a control group, which might have been a valuable addition when ana- 
lyzing the role of variables such as diabetes or family history of CAD. This is to be considered in future studies on the subject.

\section{Conclusions}

Although preliminary, this is the largest study conducted so far on Pfn1 in CAD in humans. Lower levels of serum Pfn 1 in diabetes and associations with family history of CAD and multivessel disease were shown for the first time and may suggest different regulation of its release from cells in these conditions or, possibly, a change of structure (due to a genetic mutation or post-translational modification). The role of the cytoskeleton and associated proteins is a potential new direction in the studies on atherosclerosis.

\section{Funding}

This study was supported by a research grant from the Jagiellonian University Medical College no. K/ DSC/003586 to EP.

This article was supported by the science fund of the John Paul II Hospital, Cracow, Poland (no. FN/15/2021 to E.P.).

\section{Conflict of interest}

The authors declare no conflict of interest.

\section{References}

1. Hansson GK, Libby P. The immune response in atherosclerosis: a double-edged sword. Nat Rev Immunol 2006; 6: 508-19.

2. van Steen ACl, van der Meer WJ, Hoefer IE, van Buul JD. Actin remodelling of the endothelium during transendothelial migration of leukocytes. Atherosclerosis 2020; 315: 102-10.

3. Allen A, Gau D, Roy P. The role of profilin-1 in cardiovascular diseases. J Cell Sci 2021; 134: jcs249060.

4. Paszek E, Zajdel W, Rajs T, et al. Profilin 1 and mitochondria partners in the pathogenesis of coronary artery disease? Int J Mol Sci 2021; 22: 1100.

5. Caglayan E, Romeo GR, Kappert K, et al. Profilin-1 is expressed in human atherosclerotic plaques and induces atherogenic effects on vascular smooth muscle cells. PLoS One 2010; 5: e13608.

6. Romeo GR, Moulton KS, Kazlauskas A. Attenuated expression of profilin-1 confers protection from atherosclerosis in the LDL receptor null mouse. Circ Res 2007; 101: 357-67.

7. Li Z, Zhong Q, Yang T, et al. The role of profilin-1 in endothelial cell injury induced by advanced glycation end products (AGEs). Cardiovasc Diabetol 2013; 12: 141

8. Ramaiola I, Padró T, Peña E, et al. Changes in thrombus composition and profilin-1 release in acute myocardial infarction. Eur Heart J 2015; 36: 965-75.

9. Paszek E, Żmudka K, Plens K, et al. Evaluation of profilin 1 as a biomarker in myocardial infarction. Eur Rev Med Pharmacol Sci 2020; 24: 8112-6.

10. American Diabetes Association. 5. Prevention or delay of type 2 diabetes: standards of medical care in diabetes-2018. Diabetes Care 2018; 41 (Suppl 1): S51-4.
11. Gould KL. Does coronary flow trump coronary anatomy? JACC Cardiovasc Imaging 2009; 2: 1009-23.

12. Gensini GG. A more meaningful scoring system for determining the severity of coronary heart disease. Am J Cardiol 1983; 51: 606.

13. ELISA Kit for Profilin 1 (PFN1), Sandwich ELISA, CLOUD-CLONE CORP.(CCC) [Internet]. [cited 2018 May 31]. Available from: https://www.cloud-clone.us/elisa/ELISA-Kit-for-Human-Profilin-1-(PFN1)-6179.htm

14. Romeo G, Frangioni JV, Kazlauskas A. Profilin acts downstream of LDL to mediate diabetic endothelial cell dysfunction. FASEB J 2004; 18: 725-7.

15. Hecker $M$, Wagner $A H$. Role of protein carbonylation in diabetes. J Inherit Metab Dis 2018; 41: 29-38.

16. Dalle-Donne I, Aldini G, Carini M, et al. Protein carbonylation, cellular dysfunction, and disease progression. J Cell Mol Med 2006; 10: 389-406.

17. Alkam D, Feldman EZ, Singh A, Kiaei M. Profilin1 biology and its mutation, actin(g) in disease. Cell Mol Life Sci 2017; 74: 967-81.

18. Del Poggetto E, Bemporad F, Tatini F, Chiti F. Mutations of profilin-1 associated with amyotrophic lateral sclerosis promote aggregation due to structural changes of its native state. ACS Chem Biol 2015; 10: 2553-63.

19. Boopathy S, Silvas TV, Tischbein M, et al. Structural basis for mutation-induced destabilization of profilin 1 in ALS. Proc Natl Acad Sci USA 2015; 112: 7984-9. 